\title{
Comparative Causal Analysis of the Effects of Long-Acting Muscarinic Antagonist Versus No Long-Acting Bronchodilator Use on Readmission or Mortality After Hospitalization for Chronic Obstructive Pulmonary Disease
}

\author{
Melissa H. Roberts ${ }^{1}$ (D) . Douglas W. Mapel $^{1}$ (D) Hans Petersen ${ }^{2}$
}

Published online: 2 December 2019

(c) The Author(s) 2019

\begin{abstract}
Background Retrospective observational studies may provide real-world evidence about long-acting muscarinic receptor antagonist (LAMA) effectiveness in reducing mortality or COPD-related readmission risk after a COPD hospitalization. Causal inference and competing risk statistical procedures aid in managing confounding and competing outcome events that complicate retrospective analyses.

Objective To compare COPD-related readmission and mortality risk among patients receiving a LAMA versus patients receiving no long-acting bronchodilator ("no LABD") within 30 days post-discharge.

Methods This retrospective observational analysis of patients (aged $\geq 40$ years) hospitalized for COPD used claims data (years 2004-2012). Events occurring during the period from 31 days through 12 months post-discharge were compared. The hazard ratio (HR) for the combined outcome of COPD-related readmission or mortality was estimated using Cox regression. Confounding was addressed using inverse probability of treatment weighting (IPTW). The competing risk of non-COPDrelated readmission was considered.

Results 10,405 COPD patients were included $(\mathrm{LAMA}=751$, no LABD $=9654)$. IPTW achieved a balanced sample $(10,518$ LAMA, 10,405 no LABD). Unweighted HR (LAMA vs no LABD) for COPD-related readmission or death, adjusted for age, sex, comorbidities, and baseline utilization, was 1.00 [95\% confidence interval (CI) 0.84, 1.20]. Weighted (IPTW) adjusted HR was 0.94 (95\% CI 0.88, 1.00). Unweighted and weighted HRs further adjusted for competing risk were 0.97 (95\% CI $0.82,1.16)$ and $0.91(0.86,0.98)$, respectively.

Conclusions Bias by indication and comorbidities make the measurement of retrospective COPD treatment effectiveness difficult. Using IPTW and additionally considering the competing event risk, LAMA use was associated with a small reduction in risk for COPD-related readmission or death over the period from 31 days to 12 months post-discharge.
\end{abstract}

Electronic supplementary material The online version of this article (https://doi.org/10.1007/s40801-019-00171-w) contains supplementary material, which is available to authorized users.

Melissa H. Roberts

MHRoberts@salud.unm.edu

1 College of Pharmacy, University of New Mexico, MSC09 5360, Albuquerque, NM 87131-0001, USA

2 Lovelace Respiratory Research Institute, Albuquerque, NM, USA

\section{Introduction}

Chronic obstructive pulmonary disease (COPD) was among the top ten most common principal diagnoses for nonmaternal/nonneonatal inpatient stays in the United States (US) in 2015 [1]. Importantly, individuals discharged from a hospitalization for COPD remain at high risk for another hospitalization or death. In the US, approximately one in four patients hospitalized for COPD is readmitted for COPD within one year of discharge [2-4]. Similar, if not higher, readmission rates have been reported in the United Kingdom [5, 6] and Denmark [7]. Mortality rates in the year after a COPD hospitalization have ranged from 21 to $27 \%$ [2, 4, 8-13]. 


\section{Key Points}

Real-world treatment effectiveness tends to be attenuated compared to the benefits observed in randomized clinical trials due to factors such as patient population characteristics, confounding diseases, and poor treatment adherence.

It is important to consider competing outcome events in time-to-event analyses of COPD patients due to the high prevalence of confounding illnesses.

Causal inference methods such as propensity matching and inverse probability of treatment weighting (IPTW) aid in examining causal associations between a treatment and an outcome in real-world retrospective analyses.

In this analysis, we demonstrate how these methods aid in examining the real-world effectiveness of pharmaceutical interventions at reducing COPD readmissions and death.

In 2015, the US Centers for Medicare and Medicaid Services (CMS) added COPD to the conditions in the CMS Hospital Readmissions Reduction Program (HRRP), which focuses on readmissions occurring within 30 days for any reason (all-cause) [14]. The 30-day all-cause readmission rate following a hospitalization is high, approximately $20 \%$ [15], whereas the rate for 30-day readmissions due to COPD (COPD readmissions) is much lower-approximately 5\% $[4,16]$. Since the addition of COPD to the HRRP program, most of the research into COPD hospitalization has focused on all-cause 30-day readmissions, with minimal research conducted on readmissions occurring later than 30 days after the COPD hospitalization.

COPD exacerbations are episodes in which individuals experience worsening of COPD. COPD exacerbations range from mild worsening of symptoms to acute exacerbations requiring hospitalization. Inhaled long-acting bronchodilators (LABDs) - long-acting beta-agonists (LABAs) and long-acting muscarinic receptor antagonists (LAMAs) — are recommended as maintenance therapy for COPD patients with frequent respiratory symptoms or any tendency for COPD exacerbation events [17]. Randomized clinical trials (RCTs) have demonstrated that LAMA agents (tiotropium bromide, glycopyrronium bromide, and aclidinium bromide) reduce the risk of COPD exacerbations [18]. The Global Initiative for Chronic Obstructive Lung Disease (GOLD) Global Strategy for the Diagnosis, Management, and Prevention of COPD (2011 report) recommended that patients hospitalized with an exacerbation of COPD should begin using LABDs prior to discharge [19]. However, as of 2013 in the United States, approximately one in five patients was not receiving a bronchodilator within 30 days after a COPD exacerbation-related inpatient or ED stay [20].

Tiotropium was the first LAMA to be approved; it received FDA approval in 2004 for treatment of COPD and in 2009 for the reduction of COPD exacerbations [21, 22]. The efficacy of tiotropium at reducing the risk of exacerbation-associated hospitalization was demonstrated by a pooled RCT analysis published in 2009, which showed that risk was reduced by $21 \%$ [95\% confidence interval (CI) 0.65-0.96] compared to placebo [23]. Subsequent Cochrane reviews of RCT studies for tiotropium versus placebo also found a significant risk reduction in COPD-related hospitalization (OR 0.85; 95\% CI 0.72-1.00) [24, 25], but no significant difference was found for all-cause hospitalization, or for mortality. Published RCT studies suggest similar efficacies concerning reductions in COPD hospitalization for the newer LAMA agents [26, 27].

Drug RCT studies support regulatory agency approval by demonstrating safety and efficacy. RCTs are the "gold standard," as patients are randomized to treatment groups and any confounding due to patient characteristics should be the same for both groups. However, RCTs are conducted in controlled settings and historically have not included populations that are generally representative of real-world populations. Importantly, older individuals are frequently excluded from COPD RCTs, as are individuals with asthma and many other comorbidities [28]. While there is RCT-based evidence for LAMA effectiveness in reducing exacerbation-associated hospitalization risk, LAMA effectiveness at reducing the risk of readmission or mortality after a COPD-related hospitalization in a real-world population has not been well studied.

Measuring COPD treatment effectiveness at reducing the risk of readmission due to COPD in real-world situations is challenging due to potential biases in retrospective population-based data [29, 30]. Selection biases may result in treatment cohorts that are unbalanced in important prognostic clinical factors, such as age and comorbidities. Bias by indication, the tendency for the sickest patients to get treatment, is another concern. COPD treatment may be a marker for persons with particularly severe disease, who are also more likely to have poor outcomes. Confounding characteristics related to readmissions after a COPD hospitalization include patient age, indicators of disease severity such as medications used, and comorbidities (in particular cardiovascular-related morbidities) [16, 31, 32]. Inadequate assessment and adjustment for biases can lead to false conclusions [33, 34].

Competing risks for events that preclude the event of interest are another consideration. Not all individuals in a time-to-event analysis may experience an event during the observation period. Individuals who do not experience an 
event are censored at the end of the period, as are individuals who experience an administrative event not related to the outcome of interest (e.g., health insurance disenrollment). Under this noninformative censoring, these individuals are considered to have the same risk of experiencing an event as those who are not censored [35]. However, individuals who experience an event that precludes the outcome of interest (for example death) or, in an analysis of COPD hospitalization, are hospitalized for an alternative reason do not have the same risk for the event of interest. Censoring must be handled differently for these competing risk events than for noninformative events [36].

Statistical methods exist to manage confounding and competing risk in retrospective data analyses. The use of inverse probability of treatment weighting (IPTW) the inverse of the probability of receiving the treatment actually received-is an effective adjustment method for confounding in observational studies of COPD treatment effectiveness [34, 37]. Using IPTW instead of matching results in fewer exclusions from each group and has the additional advantage of creating sample groups that are approximately the same size for statistical analyses. Similarly, methods exist for estimating time-to-event survival risk in the presence of competing events that preclude the event of interest. These are available in widely used statistical packages (SAS, Stata, and R) [36, 38]. A combination of these methods can aid in examining the question of LAMA effectiveness retrospectively. Given the high proportion of patients who do not receive a bronchodilator within 30 days of discharge, the purpose of this study was to demonstrate the use of these methods by evaluating the effectiveness of LAMA treatment dispensed within the first 30 days post-discharge at improving mortality and rehospitalization outcomes when compared to no LABD treatment among adults discharged from a COPD hospitalization.

\section{Methods}

This study performed a retrospective observational analysis comprising longitudinally collected comprehensive utilization data from US CMS fee-for-service (FFS) data for Medicare beneficiaries (Parts A, B, and D coverage), along with data from members of two US managed care plans.

\subsection{Study Population}

The Medicare data used were a 5\% sample of beneficiaries from the CMS Medicare Chronic Conditions Data Warehouse (CCW) for January 1, 2006 through December 31, 2009. Managed care claims data were from two Southwest regional managed care systems encompassing the period January 1, 2004 through December 31, 2012, and included claims for traditional commercial insurance in addition to Medicaid and Medicare (Part C) plans.

Institutional review board approval for the study was obtained through Ethical \& Independent Review Services (E\&I). This study did not involve subject recruitment and used deidentified administrative data. E\&I granted an informed consent requirement waiver and a Health Insurance Portability and Accountability Act (HIPAA) authorization requirement waiver.

\subsection{COPD Cohort}

Initial COPD hospitalizations were defined as an inpatient stay with a primary diagnosis of COPD (International Classification of Disease, 9th Revision [ICD-9] diagnosis code of 490 [bronchitis], 491.x [chronic bronchitis], 492.x [emphysema], or 496 [chronic airway obstruction, not elsewhere classified], a Diagnosis Related Group code of '088' or Medicare Severity-Diagnosis Related Group code of '190,' 191,' or ' 192 '), or a primary diagnosis of respiratory failure (ICD-9 codes 518.81-518.84) with a secondary diagnosis code of COPD (ICD-9 codes 491.x, 492.x, or 496). The earliest event meeting these criteria was the index hospitalization for the individual. The discharge date was the index date.

Included patients were aged $\geq 40$ years at the index hospitalization, and were required to have at least one COPD ICD-9 diagnosis (491.xx, 492.xx, or 496) pre-discharge or within 30 days post-discharge. The minimum index hospitalization length of stay (LOS) was 2 days, and the maximum LOS was 30 days. Patients had to be discharged to home; patients transferred to skilled nursing facilities (SNFs) or other care arrangements were excluded.

To assess comorbidities and COPD medication utilization, subjects were required to have a minimum of 90 days of health insurance coverage prior to the index hospitalization admission date, and at least 30 days of health insurance coverage after the index date. To approximate a treatment-naïve cohort, patients could not have a prescription claim for any long-acting bronchodilator (LABD) in the 90 days prior to the index hospitalization. Since this study compared LAMA use to no use, they could not have a prescription claim for another LABD treatment (a LABA or an inhaled corticosteroid (ICS)/LABA) in the 30-day post-discharge period either.

Because the 30-day post-discharge period was the exposure period reviewed for a LAMA prescription postdischarge, individuals with outcome events (see Sect. 2.4) occurring within that period were not included in the study.

\subsection{Covariates}

Demographics, comorbidities, and healthcare utilization were assessed. Baseline comorbidities were identified using the Elixhauser method [39] and the Klabunde/Deyo 
adaptation of the Charlson index [40]. Asthma, arrhythmias, and prior pneumonia diagnoses were included to further characterize patient comorbidities. Hospitalizations occurring for any reason (all-cause), hospitalizations with COPD as a secondary diagnosis, and all-cause and COPD emergency department (ED) visits in the baseline period were captured, as were COPD symptoms (dyspnea and hypoxia) and moderate exacerbations (receipt of respiratory antibiotics or oral corticosteroids [OCS] within 3 days of an office visit for COPD). Baseline COPD-related medication use (inhaled corticosteroids [ICS] and short-acting bronchodilators [SABD], including short-acting beta-agonists [SABA] and short-acting muscarinic antagonists [SAMA])) was assessed, as was the use of selected cardiovascular medications (angiotensin-converting enzyme [ACE] inhibitors, angiotensin II receptor blockers [ARBs], and beta blockers). A COPD complexity score developed by Mapel and colleagues served as a proxy for COPD severity [41].

Readmission risk factors identified by CMS were assessed using information captured during the index hospitalization [42]. Factors not utilized by CMS in risk adjustment but identified by CMS as conditions that may represent adverse outcomes of care received during admission (e.g., septicemia, nephritis) were included. Any incidence of these factors was captured under the binary variable "major index hospitalization status" (see the Data Supplement in the Electronic supplementary material, ESM).

\subsection{Outcomes}

All-cause readmissions were defined as hospitalizations for any reason. COPD-related readmissions were defined as hospitalizations in the post-discharge period that met the same diagnostic criteria as the index hospitalization, and additionally included hospitalizations with a secondary diagnosis of COPD (ICD-9 codes 491.x, 492.x, or 496). Non-COPDrelated readmissions were defined as hospitalizations not identified as being COPD-related. Mortality for CMS data observations was ascertained using date of death information contained in the dataset. Death information for managed care members was obtained from the Centers for Disease Control National Death Index.

The primary outcome was COPD-related readmission and/or death. The index date (discharge date) was the start date for observations.

\subsection{Analyses}

SAS (version 9.4) and Stata (version 14.1) statistical software were used for analyses. Binary and categorical variables are reported as numbers and percentages, and continuous variables as means and standard deviations (SDs). Comparative analyses were two-tailed and used a $p$ value of $<0.05$ to determine statistical significance. Recognizing that $p$ values are influenced by sample size, effect size (ES) - a measurement unrelated to sample size-was also calculated as a measure of the magnitude of the difference between treatment groups [43, 44]. ES was measured as the absolute standardized difference, a measure that incorporates absolute difference and variability in observations [44-47]. A standardized difference of $>0.10$ indicates a meaningful difference between groups $[47,48]$. This analysis also used Cohen's characterizations of ES above 0.10 (i.e., percentage ES: small $0.10-0.30$, medium $0.30-0.50$, and large $>0.50$; mean ES: small $0.20-0.50$, medium $0.50-0.80$, and large $>0.80$ ) [44].

Propensity to have a prescription claim for LAMA or to have no LABD prescription claim at discharge or within the first 30 days was estimated by logistic regression analysis [49]. Baseline factors with a $p$ value $<0.20$ or ES $\geq 0.04$ were included as covariates in univariate analyses. Propensity scores were used to estimate the weights (IPTW values) for individuals within each treatment group [37, 50]. IPTW values for individuals receiving LAMA were calculated as the inverse of the propensity to receive LAMA, and IPTW values for individuals with no LABD were calculated as the inverse of the propensity not to receive LAMA (i.e., 1 - [propensity to receive LAMA]).

Outcome risk, measured as a hazard ratio (HR) for the LAMA treatment group compared to the no LABD group, was estimated using Cox proportional hazards regression [51]. HR 95\% confidence intervals (95\% CI) were estimated. Subjects were followed until an event occurred, until 12 months post-index, or until the end of the analysis period (December 31, 2012), whichever occurred earliest. Subjects were administratively censored for less than 12 months of post-index follow-up time in all analyses.

Regression analyses were unweighted (IPTW values were not used) and weighted (IPTW values were used). Initial unweighted and weighted models only included sex and age as covariates. Subsequent models included all covariates (sociodemographic, baseline, and index event clinical characteristics). Additionally, competing risk of a nonCOPD-related readmission was considered in unweighted and weighted analyses using the Fine and Gray method [38], which estimates a subdistribution hazard from the cumulative incidence function after considering competing events (computed in Stata using the stcrreg function [52]). Risks estimated in this manner are similar to those estimated using Cox regression. Finally, recognizing that large weights can be a concern (for a propensity value of 0.02 , the IPTW value will be 50), we conducted a stratified analysis. A weighted stratified regression analysis was conducted to calculate effect estimates for LAMA by propensity quintile [53].

A secondary analysis assessed adherence during the postdischarge period among the LAMA group to LAMA or to 
any other LABD treatment using proportion of days covered (PDC). PDC, a ratio, was defined as the total days' supply on hand over a given period divided by the time period. Values greater than 1.00 were truncated to 1.00 .

\section{Results}

For the 2004-2012 time period, 267,471 individuals met the study inclusion criteria for COPD, and 41,345 (15.5\%) were identified with eligible hospitalizations. Over half of those with eligible hospitalizations were excluded because (1) they did not have at least 90 days of insurance coverage prior to admission, (2) they were not discharged to home, or (3) they received a prescription for a LABA or ICS/LABA combination therapy within 30 days post-discharge (see Fig. 1). Another 3545 (8.6\%) were excluded because they died or were readmitted within 30 days post-discharge $(n=3534)$ or they did not have insurance coverage for the full 30 days post-discharge, and 4318 (10.4\%) were excluded because they had a prescription for a LABD in the 90 days prior to admission. Among the 3545 excluded because of events in the 30 days post-discharge, there was no difference between the LAMA and no LABD groups for all-cause readmission ( $17.8 \%$ and $17.8 \%$, respectively), but there was a slightly higher percentage of deaths in the no LABD group $(0.6 \%$ and $1.4 \%, p<0.001$ ).

Ultimately, 10,658 individuals who had evidence of a LAMA prescription being filled $(n=751)$ or no LABD prescription being filled $(n=9907)$ within 30 days postdischarge were included. The no LABD patients comprised approximately $24.0 \%$ of the 41,345 individuals originally identified with a COPD hospitalization.

Logistic regression results for propensity to receive LAMA at discharge or within 30 days are summarized in Fig. 2. Diabetes was added after an initial model showed an effect size of close to 0.10 for diabetes (ES $0.09, p<0.0001$ ) in a weighted comparison. The C-statistic for the final propensity model was 0.66 , indicating, as shown in Fig. 3, that there was substantial overlap between groups for measured factors.

Figure 3 provides a comparative distribution summary for propensity to be prescribed LAMA. Among the no LABD group, 253 patients $(2.6 \%)$ had propensity scores lower than the lowest LAMA patient propensity score. Those patients were not comparable to any patient in the LAMA group and were not included in regression analyses. This resulted in a final count of 9654 for the no LABD group.

Table 1 (demographics and index hospitalization information) and Table 2 (baseline comorbidities, utilization, and prescription use) provide comparisons of baseline factors before and after weighting each patient according to their respective IPTW value. In unweighted comparisons, LAMA patients were younger (age 71.2 vs 72.7 years, ES 0.15 ) and less likely to be male ( $34.6 \%$ vs $37.9 \%$, ES 0.07 ). Unweighted comparisons showed no meaningful difference between groups for mean index LOS (ES 0.02), although a greater percentage of LAMA patients had mechanical ventilation (4.5\% vs $2.1 \%$, ES 0.13$)$ and a diagnosis indicating hypertensive heart and renal disease ( $7.1 \%$ vs $4.9 \%$, ES 0.09) during the index event. No substantial differences in baseline comorbidities were identified in unweighted comparisons. However, unweighted baseline comparisons provided evidence that LAMA patients may have more severe COPD. In the 90-day baseline period, a higher percentage of LAMA patients than no LABD patients (6.8\% vs $1.9 \%$, ES 0.24$)$ underwent hospitalization with a secondary COPD diagnosis, made a COPD-related ED visit (12.4\% vs $9.6 \%$, ES $0.09)$, had a moderate exacerbation $(9.6 \%$ vs $6.5 \%$, ES 0.11$)$, or had at least one SABD prescription fill (33.7\% vs $24.4 \%$, ES 0.21). In unweighted comparisons, higher percentages of LAMA patients had at least one prescription claim for an ACE inhibitor, ARB, or beta blocker. However, while there were substantive differences between groups prior to weighting, all ES estimates decreased to less than 0.10 after weighting, indicating minimal meaningful differences and demonstrating good balance between the two groups for the measured factors.

Figure 4 summarizes information by propensity score for LAMA quintile, ranging from least likely (Q1) to most likely (Q5). Shown in the accompanying table are patient numbers before weighting $(\mathrm{N})$ and after weighting $(\mathrm{WgtN})$, along with the mean, minimum, and maximum propensity scores by quintile for each treatment group. Viewed according to propensity quintiles, a greater percentage of the no LABD patients are represented in lower quintiles ( 1 and 2), and a greater percentage of the LAMA patients are in higher quintiles (4 and 5). Quintile 5 contains the greatest propensity score variance (range 0.09-0.56), and LAMA patients have a slightly higher mean propensity score within quintile 5 ( 0.148 vs 0.130$)$.

Table 3 provides comparative unweighted and weighted summaries for the period covering 31 days to 12 months post-discharge. With regard to the first event occurring postdischarge, a COPD-related readmission occurred first for a greater percentage of the LAMA group in the unweighted analysis (17.0\% vs $14.7 \%$, ES 0.15 ), but the two groups were similar after weighting (14.5\% vs $14.8 \%$, ES 0.02$)$. There was a large ES difference in deaths for the LAMA vs no LABD group, so the LAMA group had a higher unweighted percentage (18.8\% vs $17.8 \%$, ES 0.06) but a lower weighted percentage ( $16.0 \%$ vs $17.8 \%$, ES 0.11 ) for the combined outcome of COPD-related readmission or death. A non-COPD readmission occurred first for similar percentages of the treatment groups in both the unweighted and weighted analyses. 
Fig. 1 Flowchart for the selection of patients included in the study. COPD chronic obstructive pulmonary disease, $D R G$ diagnosis-related group, $F F S$ fee-for-service, ICS inhaled corticosteroid, $L A B A$ long-acting beta-agonist; $L A B D$ long-acting bronchodilator
Identification of patients age 40 or older, with prescription drug coverage, and with a minimum of two outpatient claims or one inpatient stay

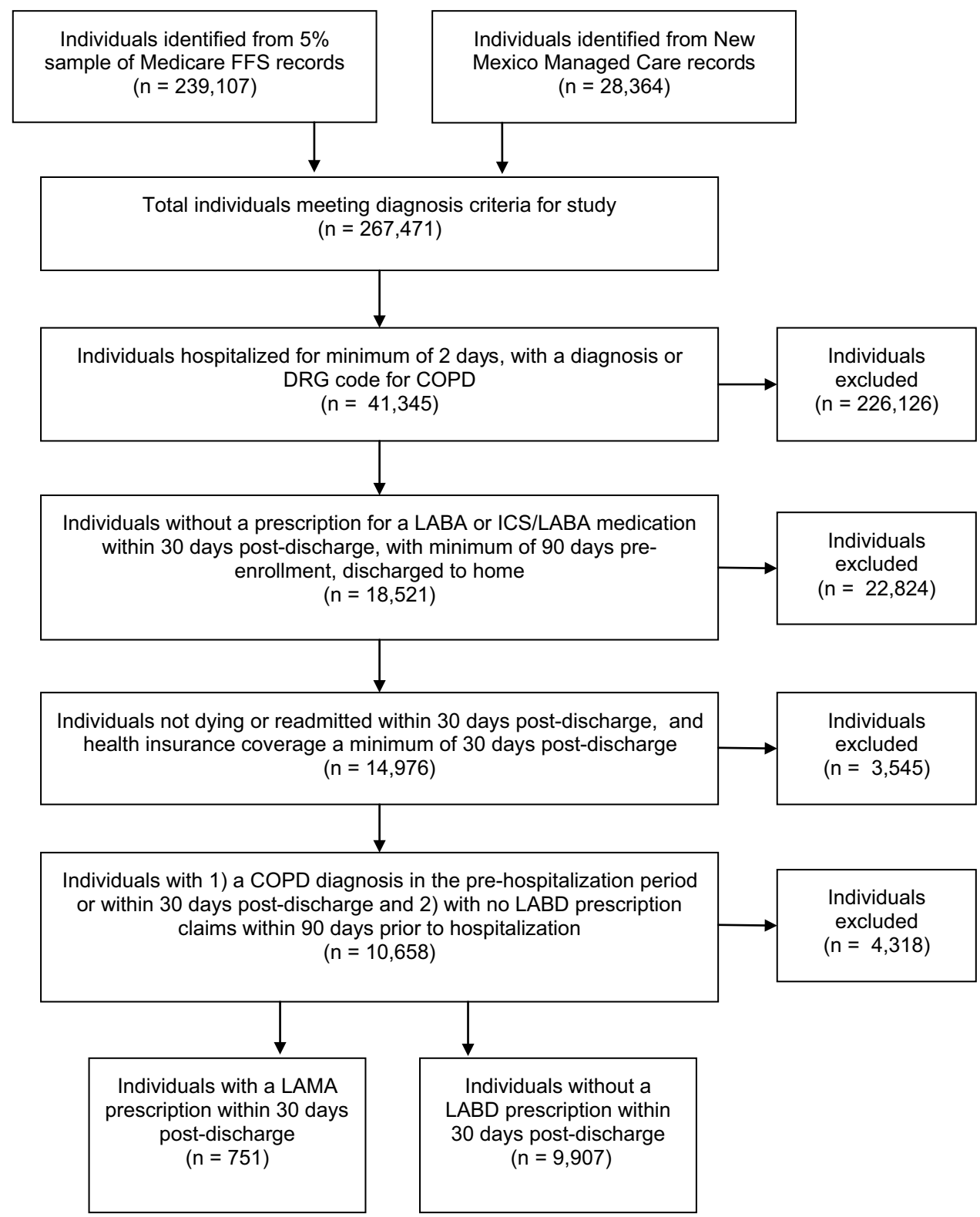

There were minimal group differences both before and after weighting for mortality or any all-cause event occurring during any point in the post-discharge period. Prior to weighting, small to large ES estimates between groups were evident for any COPD-related hospitalization, COPDrelated ED visit, or at least one moderate exacerbation. After weighting, ES estimates decreased to $<0.10$ for COPDrelated hospitalization and COPD-related ED visits, and groups were similar for these post-discharge events. Moderate differences remained between groups for post-discharge moderate exacerbations (ES range 0.42-0.57).
Figure 5 provides the cumulative incidence for the first event to occur during the post-discharge period. By the end of one year, the majority of the cohort had experienced a COPD-related or non-COPD-related readmission, or had died.

Figure 6 provides the risk estimates for the combined events of COPD-related readmission or death using unweighted, weighted, and weighted stratified models. Consideration of the competing risk for non-COPD-related readmission resulted in slightly lower HR risk estimates. There were substantive HR differences between unweighted 


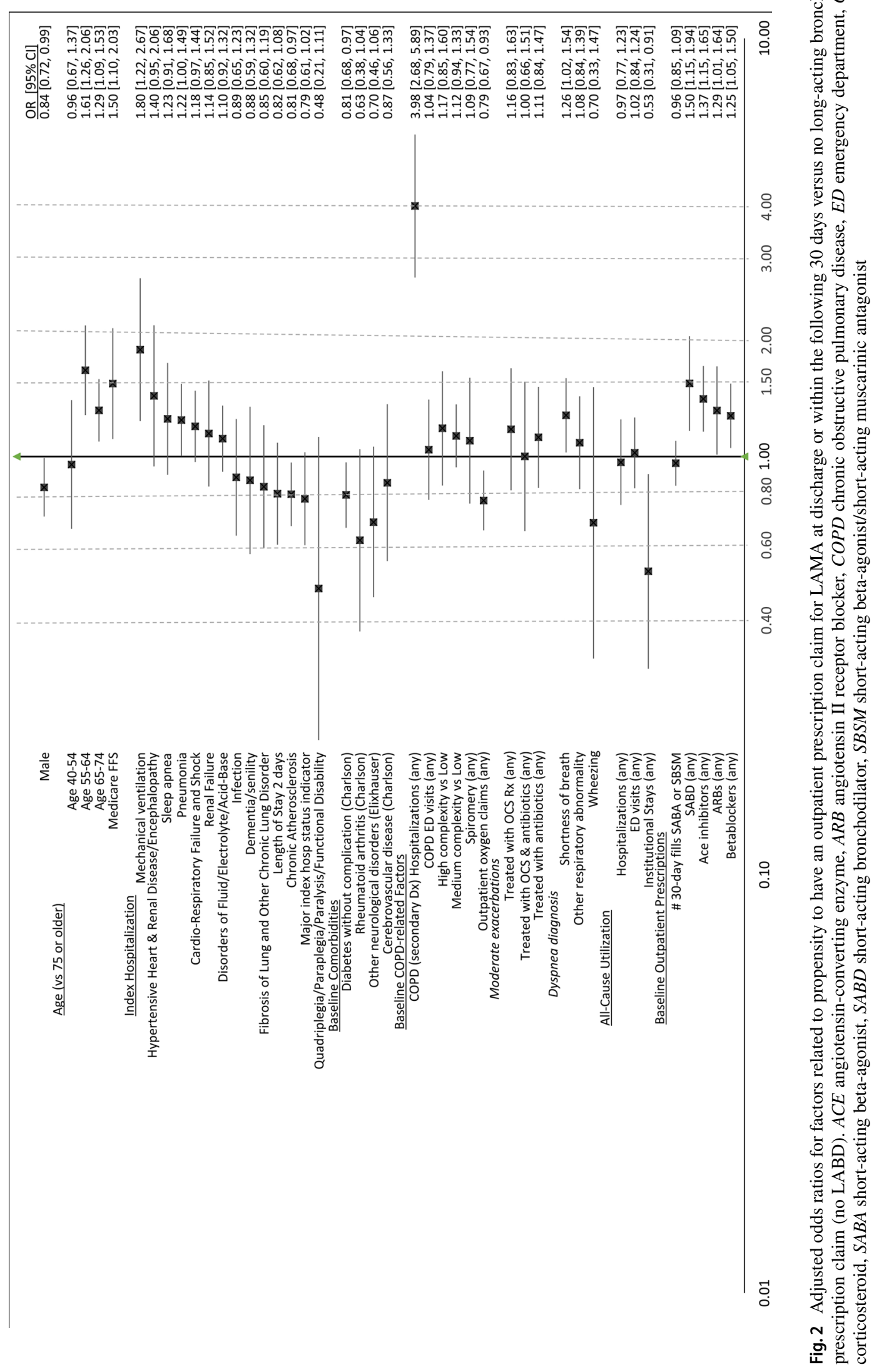


Fig. 3 Study subject distributions for propensity to have a LAMA outpatient prescription claim at discharge or within the following 30 days by treatment group (LAMA vs no LABD). The propensity regression includes information from the index hospitalization and the 90 days prior to discharge. Blue vertical lines indicate quintiles for propensity to have a claim for LAMA at index. By definition, $20 \%$ of the entire cohort is within each quintile, but the distribution of LAMA vs no $L A B D$ varies within each quintile. $L A B D$ long-acting bronchodilator, LAMA longacting muscarinic antagonist

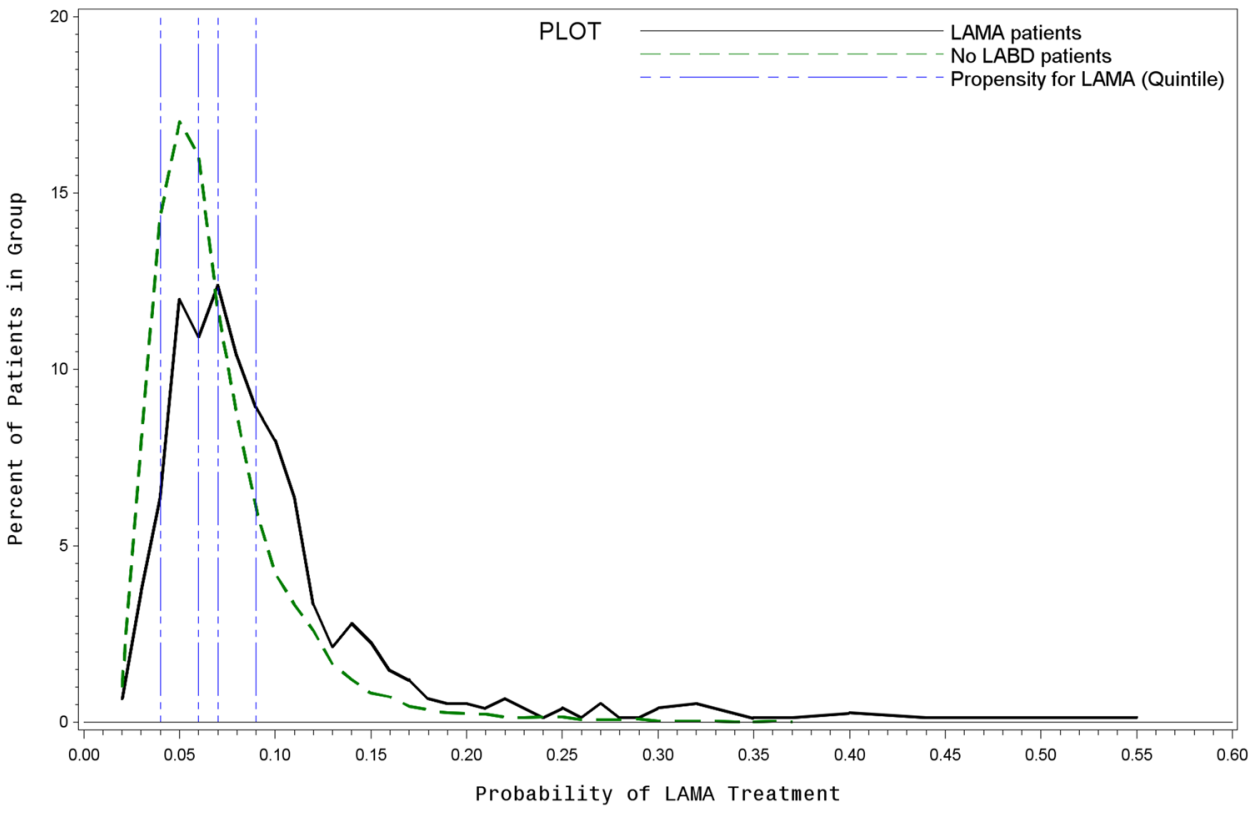

and weighted regression models. Larger sample sizes in the weighted models resulted in narrower estimated confidence intervals. Without incorporating the IPTW values, the estimate for the LAMA effect after adjusting for all baseline covariates was 1.00 (95\% CI 0.84, 1.20), and it was 0.97 (95\% CI 0.82, 1.16) after accounting for competing risk. Alternatively, in the weighted model, the fully adjusted HR estimate was 0.94 (95\% CI $0.88,1.00)$, and it was $0.91(95 \%$ CI $0.86,0.98$ ) after accounting for competing risk. The stratified weighted model suggests that the HR for LAMA estimated in the prior weighted models is driven by the lowestpropensity quintile. The HR estimate for LAMA compared to no LABD was significantly greater than 1.0 for the fifth and highest-propensity quintile. Individuals in quintile 5 had the highest prevalence of serious conditions during the index hospitalization, including mechanical ventilation $(9.0 \%)$, cardiorespiratory failure and shock (26.6\%), pneumonia (25.6\%), hypertensive heart and kidney disease (12.6\%), and renal failure (19.9\%) (data not shown). The fifth quintile also had the highest prevalence of baseline clinical factors, including pneumonia (25.0\%), recent COPD-related ED visits $(16.3 \%)$ or hospitalizations $(10.8 \%)$, moderate AECOPD requiring steroids or antibiotics $(15.1 \%)$, prescription fill for SABDs within 90 days (55.0\%), and any use of ACE inhibitors $(42.8 \%)$, ARBs (17.4\%), and beta blockers (43.8\%).

Among the LAMA treatment group, mean (SD) persistence with LAMA therapy during the first 90 and 180 days post-discharge as measured using PDC was 0.615 (0.252) and 0.500 (0.292), respectively. During 270 days and 12 months post-discharge, PDC was 0.429 (0.306) and 0.396 (0.308), respectively (data not shown). PDC for any LABD was only slightly higher. During the first 180 days, mean
PDC was similar for patients with a COPD-related readmission, those who died, and those who did not have any event, censored for a competing event or censored for loss to follow-up. In the last half of the year, mean PDC values for patients with a COPD-related readmission were approximately 0.10 lower.

\section{Discussion}

In this retrospective analysis of US Medicare and managed care data from 2004 to 2012, a prescription for an inhaled LAMA within 30 days after discharge from a hospitalization for COPD significantly reduced the risk for the combined endpoint of either death or COPD-related rehospitalization in the period from 31 days to 12 months after discharge as compared to patients not prescribed any LABD therapy. As expected, there was potential confounding from comorbidities and selection biases. In unweighted analyses, higher percentages of the patients prescribed LAMA after discharge had markers of unstable COPD: increased number of COPDrelated hospitalizations, moderate exacerbations, and higher utilization of SABD respiratory medications at baseline. In unweighted regression models that only adjusted for age and sex, a LAMA prescription was associated with a higher, but not significantly higher, HR risk $(1.09,95 \%$ CI $0.92,1.29)$ for the combined endpoint. IPTW was used to adjust for confounding, and in weighted regression models adjusting for age and sex, a statistically significant, albeit reduced, HR of 0.90 (95\% CI 0.85, 0.97) was obtained. After further considering all covariates and the competing risk of non-COPD-related readmission, the weighted HR for the 
Table 1 Demographics, baseline comorbidities, and utilization (unweighted and weighted) by discharge treatment

\begin{tabular}{|c|c|c|c|c|c|c|c|c|}
\hline & \multicolumn{4}{|c|}{ Unweighted } & \multicolumn{4}{|l|}{ Weighted } \\
\hline & LAMA & No LABD & ES & $P$ value & LAMA & No LABD & ES & $P$ value \\
\hline Number of individuals & 751 & 9654 & & & 10,518 & 10,402 & & \\
\hline Male, $\%$ & 34.6 & 37.9 & 0.07 & 0.07 & 37.4 & 37.7 & 0.01 & 0.71 \\
\hline Age, mean (SD) & $71.2(9.7)$ & $72.7(10.3)$ & 0.15 & 0.0001 & $71.9(10.3)$ & $72.6(10.6)$ & 0.07 & 0.07 \\
\hline Age $40-54, \%$ & 5.5 & 6.3 & 0.03 & 0.37 & 7.3 & 6.2 & 0.04 & 0.002 \\
\hline Age $55-64, \%$ & 14.2 & 9.8 & 0.14 & $<0.0001$ & 10.8 & 10.1 & 0.02 & 0.11 \\
\hline Age $65-74, \%$ & 42.7 & 39.1 & 0.08 & 0.05 & 37.8 & 39.3 & 0.03 & 0.03 \\
\hline Age $\geq 75, \%$ & 37.5 & 44.9 & 0.15 & $<0.0001$ & 44.1 & 44.3 & 0.01 & 0.70 \\
\hline Emphysema, \% & 8.4 & 7.7 & 0.03 & 0.47 & 8.1 & 7.7 & 0.02 & 0.25 \\
\hline \multicolumn{9}{|l|}{ Index hospitalization } \\
\hline Length of stay (days), mean (SD) & $5.3(2.9)$ & $5.2(3.2)$ & 0.02 & 0.54 & $5.1(2.8)$ & $5.2(3.3)$ & 0.04 & 0.25 \\
\hline Length of stay $=2$ days, $\%$ & 7.9 & 9.6 & 0.06 & 0.11 & 10.1 & 9.5 & 0.02 & 0.12 \\
\hline Mechanical ventilation, $\%$ & 4.5 & 2.1 & 0.13 & $<0.0001$ & 2.4 & 2.3 & 0.01 & 0.62 \\
\hline \multicolumn{9}{|l|}{ Comorbidities noted at index, $\%$} \\
\hline \multicolumn{9}{|l|}{ Cardiovascular/respiratory } \\
\hline Arrhythmias & 20.1 & 20.6 & 0.01 & 0.76 & 20.1 & 20.5 & 0.01 & 0.44 \\
\hline Chronic atherosclerosis & 27.4 & 30.0 & 0.06 & 0.14 & 29.3 & 29.8 & 0.01 & 0.46 \\
\hline Congestive heart failure & 30.5 & 28.6 & 0.04 & 0.26 & 29.3 & 28.7 & 0.02 & 0.27 \\
\hline Cardiorespiratory fail/shock & 21.7 & 19.3 & 0.06 & 0.12 & 19.7 & 19.5 & 0.003 & 0.81 \\
\hline Pulmonary fibrosis/other chronic lung disease & 5.2 & 6.4 & 0.05 & 0.19 & 6.0 & 6.3 & 0.01 & 0.31 \\
\hline Pneumonia & 19.0 & 16.4 & 0.07 & 0.07 & 16.9 & 16.6 & 0.01 & 0.57 \\
\hline Vascular/circulatory disease & 11.6 & 10.6 & 0.03 & 0.40 & 11.8 & 10.6 & 0.04 & 0.005 \\
\hline \multicolumn{9}{|l|}{ Other noted conditions } \\
\hline Major index hospitalization status & 10.1 & 12.3 & 0.07 & 0.08 & 12.8 & 12.1 & 0.02 & 0.16 \\
\hline Sleep apnea & 7.1 & 5.6 & 0.06 & 0.10 & 5.6 & 5.7 & 0.004 & 0.80 \\
\hline Depression & 10.3 & 9.5 & 0.02 & 0.51 & 10.0 & 9.5 & 0.02 & 0.22 \\
\hline Diabetes and DM complications & 26.8 & 27.0 & 0.005 & 0.90 & 28.0 & 26.9 & 0.02 & 0.07 \\
\hline Dementia or senility & 3.7 & 4.9 & 0.06 & 0.14 & 5.4 & 4.8 & 0.03 & 0.05 \\
\hline Fluid/electrolyte/acid-base disorders & 23.2 & 21.1 & 0.05 & 0.19 & 21.2 & 21.3 & 0.002 & 0.89 \\
\hline History of infection & 5.9 & 7.0 & 0.05 & 0.25 & 7.2 & 6.9 & 0.01 & 0.33 \\
\hline Hypertensive heart and renal disease & 7.1 & 4.9 & 0.09 & 0.01 & 5.2 & 5.1 & 0.01 & 0.67 \\
\hline Major psychiatric disorders & 3.6 & 3.4 & 0.01 & 0.76 & 3.7 & 3.4 & 0.02 & 0.20 \\
\hline Iron deficiencies/other unspecified anemia & 13.0 & 14.5 & 0.04 & 0.28 & 13.2 & 14.5 & 0.04 & 0.004 \\
\hline Other gastrointestinal disorders & 25.0 & 24.8 & 0.004 & 0.91 & 25.0 & 24.9 & 0.002 & 0.87 \\
\hline Other cancers & 2.9 & 2.6 & 0.02 & 0.61 & 3.4 & 2.6 & 0.05 & 0.001 \\
\hline Other psychiatric disorders & 7.5 & 7.2 & 0.01 & 0.77 & 6.4 & 7.2 & 0.03 & 0.02 \\
\hline Quadriplegia and other functional disability & 0.8 & 1.3 & 0.05 & 0.22 & 1.5 & 1.3 & 0.02 & 0.27 \\
\hline Renal failure & 13.7 & 11.4 & 0.07 & 0.06 & 11.6 & 11.6 & 0.001 & 0.92 \\
\hline
\end{tabular}

$D M$ diabetes mellitus, $E S$ effect size (absolute standardized difference), $S D$ standard deviation

combined outcome of death or COPD-related rehospitalization was 0.91 (95\% CI $0.86,0.98)$, significantly favoring LAMA treatment. This result is similar to the Cochrane review odds ratio estimate for COPD-related hospitalization for tiotropium vs placebo $(0.85 ; 95 \%$ CI $0.72-1.00)$ [24, 25]. A stratified analysis, however, indicates that some residual unmeasured confounding may still exist, as the HR varied across the propensity for LAMA quintiles from a HR of 0.79 among those least likely to receive LAMA to a HR of 1.17 among those most likely to receive LAMA.

In this predominantly Medicare population, for the period 2004-2012, approximately $25 \%$ of patients discharged from a hospitalization for COPD did not receive any LABD. Baker and colleagues similarly found a high percentage (26\%) who did not have a prescription claim for a LABD or SABD both 90 days before and after a hospitalization 
Table 2 Baseline comorbidities and utilization (unweighted and weighted) by discharge treatment

\begin{tabular}{|c|c|c|c|c|c|c|c|c|}
\hline & \multicolumn{4}{|c|}{ Unweighted } & \multicolumn{4}{|l|}{ Weighted } \\
\hline & LAMA & No LABD & ES & $P$ value & LAMA & No LABD & ES & $P$ value \\
\hline Number of individuals & 751 & 9654 & & & 10,518 & 10,402 & & \\
\hline \multicolumn{9}{|l|}{ Charlson, \% } \\
\hline Charlson comorbidity score, mean (SD) & $2.0(1.6)$ & $2.0(1.6)$ & 0.01 & 0.85 & $2.1(1.7)$ & $2.0(1.7)$ & 0.03 & 0.45 \\
\hline Myocardial infarction & 5.1 & 4.4 & 0.03 & 0.41 & 4.6 & 4.4 & 0.01 & 0.45 \\
\hline $\mathrm{CHF}$ & 27.7 & 26.5 & 0.03 & 0.48 & 26.7 & 26.6 & 0.003 & 0.82 \\
\hline Peripheral vascular disease & 6.7 & 7.5 & 0.03 & 0.39 & 7.1 & 7.5 & 0.01 & 0.29 \\
\hline Cerebrovascular disease & 3.3 & 4.1 & 0.04 & 0.29 & 4.4 & 4.1 & 0.02 & 0.18 \\
\hline COPD & 86.2 & 85.5 & 0.02 & 0.63 & 84.9 & 85.6 & 0.02 & 0.14 \\
\hline Rheumatoid arthritis & 2.3 & 3.0 & 0.05 & 0.23 & 3.4 & 3.0 & 0.02 & 0.12 \\
\hline Diabetes & 26.5 & 27.2 & 0.02 & 0.67 & 27.7 & 27.2 & 0.01 & 0.37 \\
\hline Diabetes with sequelae & 4.0 & 3.7 & 0.01 & 0.73 & 4.3 & 3.7 & 0.03 & 0.04 \\
\hline Renal disease & 7.9 & 7.2 & 0.03 & 0.50 & 6.9 & 7.3 & 0.02 & 0.24 \\
\hline Cancer & 7.1 & 6.8 & 0.01 & 0.80 & 7.5 & 6.8 & 0.03 & 0.06 \\
\hline \multicolumn{9}{|l|}{ Elixhauser, $\%^{\mathrm{a}}$} \\
\hline Valvular disease & 4.4 & 4.8 & 0.02 & 0.58 & 4.7 & 4.8 & 0.01 & 0.69 \\
\hline Pulmonary circular disease & 4.9 & 4.5 & 0.02 & 0.62 & 4.5 & 4.5 & 0.0004 & 0.98 \\
\hline Other neurological disorders & 3.6 & 4.7 & 0.06 & 0.15 & 5.4 & 4.6 & 0.04 & 0.01 \\
\hline Hypothyroidism & 9.9 & 9.1 & 0.03 & 0.46 & 9.5 & 9.1 & 0.01 & 0.31 \\
\hline Obesity & 4.5 & 4.5 & 0.004 & 0.93 & 4.3 & 4.5 & 0.01 & 0.56 \\
\hline Fluid and electrolyte disorders & 14.6 & 13.3 & 0.04 & 0.31 & 12.4 & 13.4 & 0.03 & 0.03 \\
\hline Deficiency anemias & 12.0 & 10.9 & 0.03 & 0.35 & 11.5 & 10.9 & 0.02 & 0.20 \\
\hline Depression & 7.1 & 6.4 & 0.03 & 0.49 & 7.3 & 6.4 & 0.03 & 0.02 \\
\hline Psychoses & 2.5 & 3.0 & 0.03 & 0.45 & 2.8 & 3.0 & 0.01 & 0.37 \\
\hline \multicolumn{9}{|l|}{ Other comorbidities, $\%$} \\
\hline Asthma & 16.6 & 16.6 & 0.003 & 0.95 & 15.2 & 16.7 & 0.04 & 0.004 \\
\hline Arrhythmia & 20.1 & 19.0 & 0.03 & 0.46 & 20.1 & 19.0 & 0.03 & 0.04 \\
\hline Pneumonia & 20.6 & 18.1 & 0.07 & 0.08 & 20.5 & 18.2 & 0.06 & $<0.0001$ \\
\hline Hypoxia & 15.2 & 14.7 & 0.01 & 0.73 & 15.5 & 14.7 & 0.02 & 0.11 \\
\hline \multicolumn{9}{|l|}{ Mapel complexity index } \\
\hline Overall score, mean (SD) & $1.7(0.6)$ & $1.7(0.6)$ & 0.08 & 0.04 & $1.7(0.6)$ & $1.7(0.6)$ & 0.01 & 0.70 \\
\hline Low (1), $\%$ & 33.4 & 37.6 & 0.09 & 0.02 & 36.3 & 37.3 & 0.02 & 0.12 \\
\hline Medium (2), \% & 58.5 & 54.7 & 0.08 & 0.05 & 56.2 & 55.0 & 0.02 & 0.08 \\
\hline High (3), \% & 8.1 & 7.7 & 0.02 & 0.65 & 7.5 & 7.7 & 0.01 & 0.63 \\
\hline \multicolumn{9}{|l|}{ COPD-related utilization, $\%$} \\
\hline Hospitalizations (secondary diagnosis of COPD) & 6.8 & 1.9 & 0.24 & $<0.0001$ & 2.2 & 2.3 & 0.004 & 0.80 \\
\hline ED visits & 12.4 & 9.6 & 0.09 & 0.01 & 9.6 & 9.8 & 0.01 & 0.56 \\
\hline \multicolumn{9}{|l|}{ Moderate exacerbations } \\
\hline With OCS Rx & 7.3 & 4.7 & 0.11 & 0.001 & 5.1 & 4.9 & 0.01 & 0.38 \\
\hline With antibiotic $\mathrm{Rx}$ & 10.1 & 7.5 & 0.09 & 0.01 & 7.8 & 7.7 & 0.003 & 0.83 \\
\hline With OCS and antibiotic Rx (same day) & 5.2 & 3.4 & 0.09 & 0.01 & 3.9 & 3.5 & 0.02 & 0.15 \\
\hline Any OCS or OCS/antibiotic exacerbation & 9.6 & 6.5 & 0.11 & 0.001 & 7.0 & 6.7 & 0.01 & 0.47 \\
\hline Spirometry procedure & 5.2 & 4.5 & 0.03 & 0.37 & 4.5 & 4.5 & 0.003 & 0.84 \\
\hline Outpatient oxygen & 35.4 & 37.3 & 0.04 & 0.30 & 37.4 & 37.2 & 0.005 & 0.72 \\
\hline Any outpatient visit for dyspnea & 28.6 & 24.5 & 0.09 & 0.01 & 26.2 & 24.8 & 0.03 & 0.02 \\
\hline Shortness of breath & 23.6 & 19.1 & 0.11 & 0.003 & 20.2 & 19.4 & 0.02 & 0.19 \\
\hline Wheezing & 1.1 & 1.2 & 0.01 & 0.70 & 1.3 & 1.2 & 0.01 & 0.40 \\
\hline Respiratory abnormality, other & 12.9 & 10.7 & 0.07 & 0.06 & 12.9 & 10.9 & 0.06 & $<0.0001$ \\
\hline
\end{tabular}


Table 2 (continued)

\begin{tabular}{|c|c|c|c|c|c|c|c|c|}
\hline & \multicolumn{4}{|c|}{ Unweighted } & \multicolumn{4}{|l|}{ Weighted } \\
\hline & LAMA & No LABD & ES & $P$ value & LAMA & No LABD & ES & $P$ value \\
\hline \multicolumn{9}{|l|}{ All-cause encounters, $\%$} \\
\hline Hospitalizations & 21.3 & 17.2 & 0.10 & 0.004 & 18.2 & 17.5 & 0.02 & 0.17 \\
\hline ER visits & 35.7 & 31.9 & 0.08 & 0.03 & 32.8 & 32.2 & 0.01 & 0.36 \\
\hline Institutional stays & 2.3 & 2.4 & 0.01 & 0.76 & 2.9 & 2.4 & 0.03 & 0.05 \\
\hline \multicolumn{9}{|l|}{ COPD medication, any use, $\%$} \\
\hline Any SABD & 33.7 & 24.4 & 0.21 & $<0.0001$ & 26.3 & 25.1 & 0.03 & 0.06 \\
\hline SABA & 23.8 & 17.6 & 0.16 & $<0.0001$ & 18.7 & 18.1 & 0.02 & 0.26 \\
\hline SABA/SAMA & 11.1 & 8.2 & 0.10 & 0.01 & 8.7 & 8.5 & 0.01 & 0.55 \\
\hline SAMA & 3.2 & 2.5 & 0.04 & 0.27 & 2.4 & 2.6 & 0.01 & 0.48 \\
\hline $\begin{array}{l}\text { No. of 30-day prescription claims, SABA or } \\
\text { SABA/SAMA, mean (SD) }\end{array}$ & $0.5(1.0)$ & $0.4(0.9)$ & 0.15 & 0.0002 & $0.4(0.9)$ & $0.4(0.9)$ & 0.01 & 0.78 \\
\hline Any ICS prescription claim, $\%$ & 4.8 & 4.5 & 0.02 & 0.66 & 4.2 & 4.5 & 0.02 & 0.18 \\
\hline \multicolumn{9}{|c|}{ Cardiovascular medication, any prescription claim, $\%$} \\
\hline ACE inhibitors & 27.6 & 21.1 & 0.15 & $<0.0001$ & 21.9 & 21.6 & 0.01 & 0.60 \\
\hline ARBs & 12.0 & 9.5 & 0.08 & 0.03 & 10.9 & 9.7 & 0.04 & 0.004 \\
\hline Beta-blockers & 30.4 & 24.6 & 0.13 & 0.0004 & 26.3 & 25.1 & 0.03 & 0.05 \\
\hline
\end{tabular}

$A C E$ angiotensin-converting enzyme, $A R B$ angiotensin II receptor blocker, $C H F$ congestive heart failure, $C O P D$ chronic obstructive pulmonary disease, $D M$ diabetes mellitus, $E D$ emergency department, $E S$ effect size (absolute standardized difference), $O C S$ oral corticosteroid, $R x$ prescription, $S A B A$ short-acting beta-agonist, $S A B D$ short-acting bronchodilator, SAMA short-acting muscarinic antagonist, $S D$ standard deviation

${ }^{a}$ Conditions not captured by Charlson comorbidities are shown

Fig. 4 Box plots and descriptive statistics, by quintile and by treatment group (LAMA vs no LABD), for propensity to receive LAMA treatment. Quintile $1(\mathrm{Q} 1)$ is the lowest-propensity quintile and quintile 5 (Q5) is the highest. $N$ actual number of observations for each group. $W g t N$ weighted $\mathrm{N}$ for the IPTW analyses. Dot and plus symbols indicate mean values. $L A B D$ long-acting bronchodilator, LAMA long-acting muscarinic antagonist

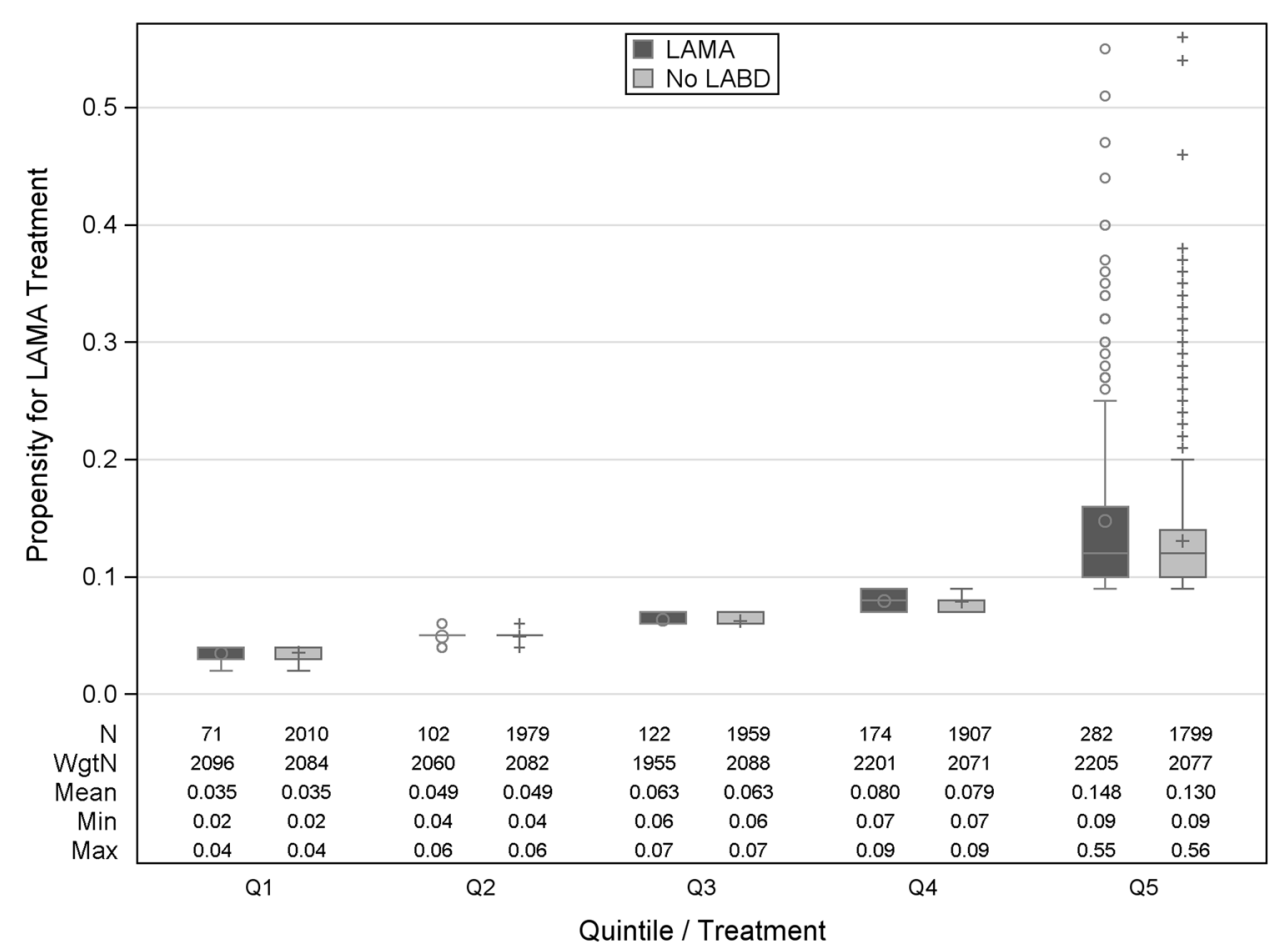

for COPD, based on an analysis of commercial insurance and the Medicare Supplement for the period 2009 through 2011 [54]. While our data are over 5 years old, according to 2017 US Healthcare Effectiveness Data and Information Set
(HEDIS) statistics, nonreceipt of a bronchodilator remains a concern, as no bronchodilator was prescribed for $20 \%$ of acute inpatient discharges or ED encounters among commercial, Medicaid, and Medicare HMO members $\geq 40$ years 
Table 3 Post-discharge utilization ${ }^{\mathrm{a}}$ (unweighted and weighted) by discharge treatment

\begin{tabular}{|c|c|c|c|c|c|c|c|c|}
\hline & \multicolumn{4}{|c|}{ Unweighted } & \multicolumn{4}{|c|}{ Weighted } \\
\hline & LAMA & No LABD & ES & $P$ value & LAMA & No LABD & ES & $P$ value \\
\hline Number of individuals & 751 & 9654 & & & 10,518 & 10,402 & & \\
\hline \multicolumn{9}{|l|}{ Earliest event ( 31 days to 12 months), $\%$} \\
\hline Non-COPD-related readmission & 33.2 & 32.0 & 0.03 & 0.53 & 32.9 & 32.0 & 0.03 & 0.19 \\
\hline COPD-related readmission & 17.0 & 14.7 & 0.15 & 0.09 & 14.5 & 14.8 & 0.02 & 0.53 \\
\hline Death & 1.7 & 3.0 & 0.68 & 0.04 & 1.5 & 3.0 & 0.83 & $<0.0001$ \\
\hline COPD-related readmission or death & 18.8 & 17.8 & 0.06 & 0.49 & 16.0 & 17.8 & 0.11 & $<0.001$ \\
\hline \multicolumn{9}{|l|}{ All events ( 31 days to 12 months), $\%$} \\
\hline Death post-discharge & 11.2 & 12.0 & 0.08 & 0.49 & 11.3 & 12.0 & 0.06 & 0.11 \\
\hline \multicolumn{9}{|l|}{ All-cause utilization } \\
\hline Hospitalizations & 51.5 & 49.0 & 0.05 & 0.28 & 49.6 & 49.1 & 0.01 & 0.52 \\
\hline ED visits & 57.3 & 55.3 & 0.03 & 0.30 & 54.9 & 55.4 & 0.01 & 0.48 \\
\hline \multicolumn{9}{|l|}{ COPD-related utilization } \\
\hline Hospitalizations & 23.4 & 19.6 & 0.18 & 0.01 & 20.7 & 19.7 & 0.05 & 0.09 \\
\hline ED visits & 20.9 & 18.0 & 0.15 & 0.05 & 19.3 & 18.0 & 0.07 & 0.02 \\
\hline \multicolumn{9}{|l|}{ Moderate exacerbations } \\
\hline With OCS Rx & 22.1 & 11.3 & 0.63 & $<0.0001$ & 20.7 & 11.4 & 0.57 & $<0.0001$ \\
\hline With antibiotic Rx & 27.6 & 16.4 & 0.51 & $<0.0001$ & 26.5 & 16.5 & 0.47 & $<0.0001$ \\
\hline With OCS and antibiotic Rx (same day) & 17.8 & 10.5 & 0.52 & $<0.0001$ & 16.3 & 10.6 & 0.42 & $<0.0001$ \\
\hline
\end{tabular}

$C O P D$ chronic obstructive pulmonary disease, $E D$ emergency room, $E S$ effect size (absolute standardized difference), $O C S$ oral corticosteroid, $R x$ prescription, $S D$ standard deviation

${ }^{a}$ Events/utilization occurring from after 30 days to one-year post-discharge

Fig. 5 Cumulative incidence for first event post-discharge among COPD-related readmission, non-COPD-related readmission, and death. $C O P D$ chronic obstructive pulmonary disease

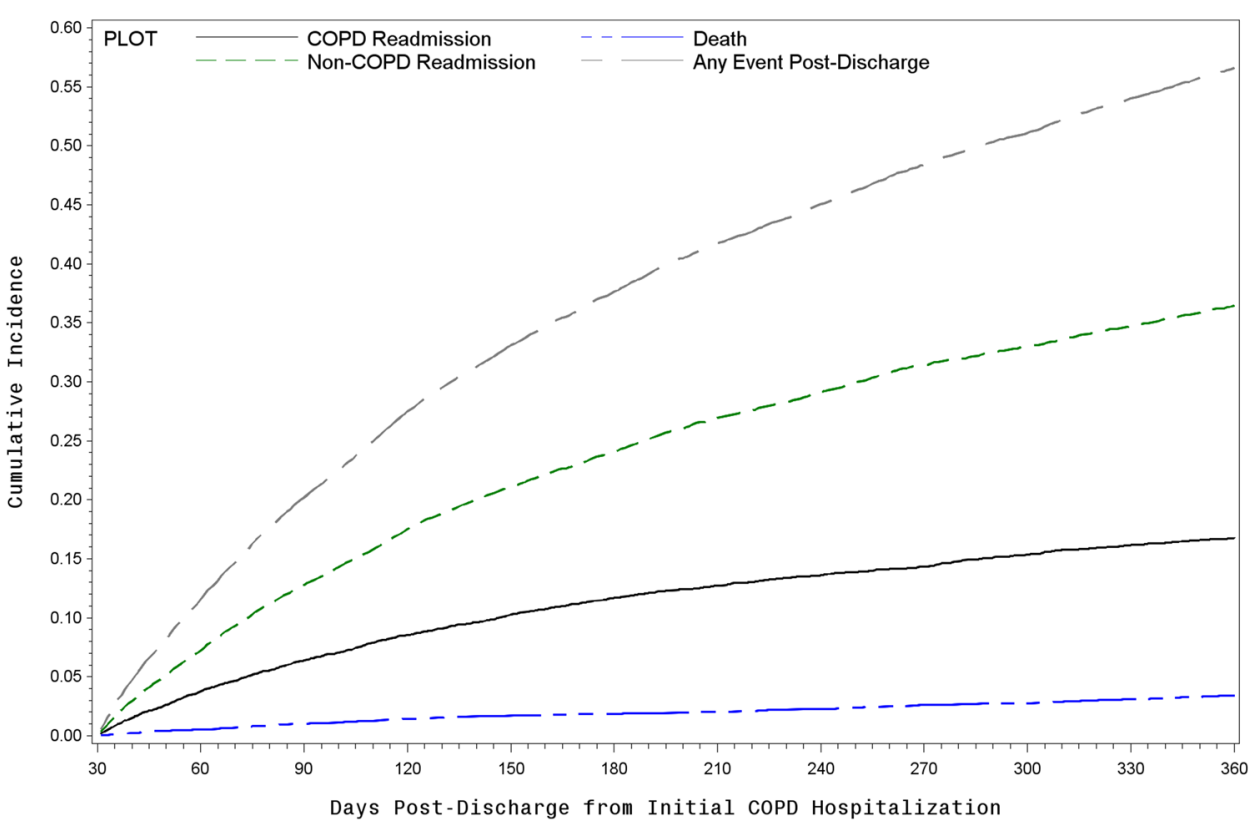

old [20]. Among PPO members, the percentage was slightly higher [20].

In our study population, approximately $12 \%$ died in the year after hospitalization, and 50\% were readmitted for any cause. These rates are lower than the $26 \%$ mortality rate and
$64 \%$ readmission rate reported by Lindenauer and colleagues based on Medicare data for 2009-2014 [13]. However, their study population had a higher percentage of male patients and patients with congestive heart failure, diabetes, and renal failure, and included patients originally discharged to 


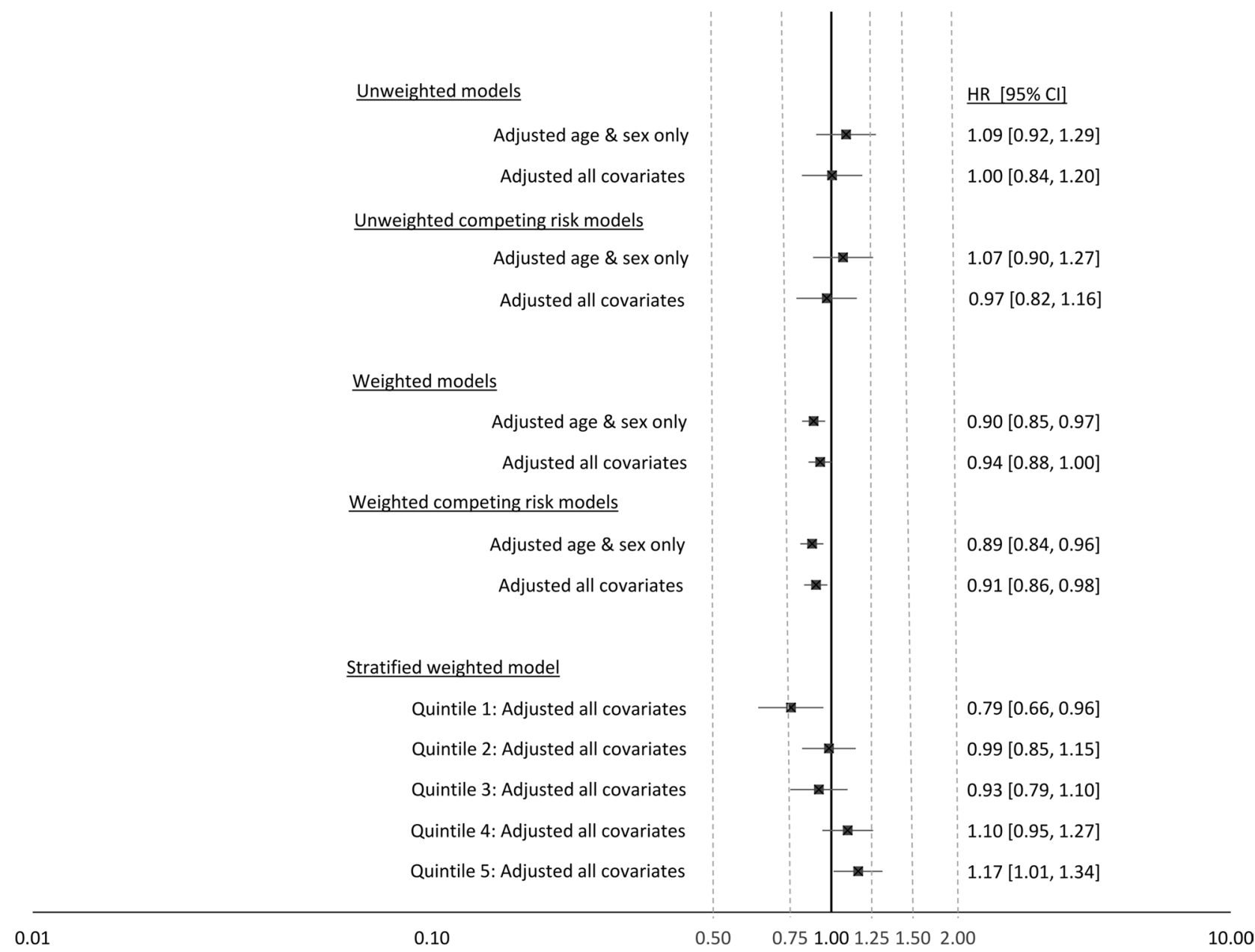

Fig. 6 Cox regression model effect estimates for COPD-related readmission or death. Models are unweighted and weighted (using IPTW values). HR is for LAMA compared to no LABD treatment. Competing risk models consider the competing risk of non-COPD-related

SNFs [13]. The average index admission LOS in our analysis was 5.1 days. This LOS was similar to the average LOS of 4.9 days for an inpatient with a first-listed discharge reason of COPD in the 2012 National Heart Lung and Blood Institute Chartbook [55].

Few studies have examined the comparative effectivenesses of LAMA and no LABD treatment at reducing hospital readmission. In analyses of Medicare recipients for 1997-2005, use of COPD maintenance drugs was associated with a reduced risk of hospitalization (HR 95\% CI $0.61,0.79)$ and readmission within 30 days (OR 95\% CI $0.63,0.87$ ) [56]. Another study used the IPTW analysis method to retrospectively examine outcomes up to 30 days post-discharge from a hospitalization occurring January 2010 through June 2011 in which LABD treatment was provided during the hospitalization [57]. No association was found between LABD treatment and improved 30-day readmission. The stratified weighted model provides an estimated HR [95\% CI] by quintile for propensity for LAMA treatment (quintile 1 is the lowest and quintile 5 is the highest). $C I$ confidence interval, $H R$ hazard ratio

readmission and initial hospitalization LOS [57]. Our study instead examined associations between LAMA prescribed as a maintenance therapy within 30 days post-discharge and outcomes occurring after those 30 days, and it did find a small but statistically significant risk reduction.

Our study compared LAMA use to no LABD therapy. Other LABD therapies (LABA and combination ICS/ LABA) were not included. Comparative effectiveness studies comparing LAMA to other LABD medications in COPD patients at risk for exacerbations suggest other classes of LABD treatments have similar or greater efficacy when compared to no LABD therapy. One study from Italy compared survival among patients prescribed tiotropium or LABAs after a COPD hospitalization using both intent-to-treat and as-treated analysis, and found no substantial differences [58]. Another study comparing the effectiveness of budesonide/ formoterol, an ICS/LABA combination, and tiotropium 
bromide among COPD patients new to these treatments found that time to exacerbation was substantially reduced among budesonide/formoterol users (HR 95\% CI 0.70, 0.87) after applying propensity score matching techniques [59]. A recent Cochrane study that reviewed RCTs comparing LABDs found no difference in mortality risk for patients treated with LAMA therapy compared to either LABA/ LAMA or to ICS/LABA combination therapy, nor any difference in severe exacerbation risk for LAMA therapy compared to either LABA/LAMA or ICS/LABA combination therapy [60]. The LAMA therapies considered in the review included tiotropium bromide, aclidinium, umeclidinium, and glycopyrrolate [60].

When our weighted model results were stratified by quintile for propensity for LAMA treatment, the results were not equal; patients with the lowest propensity to receive treatment were more likely to have better treatment outcomes. This finding may seem paradoxical, but may be explained by indication bias: patients with more severe COPD are more likely to receive treatment, particularly those fitting a "frequent exacerbator" phenotype. Even though LAMA treatment may be beneficial among the most unstable COPD patients, it does not completely neutralize their risk for exacerbation. In this retrospective analysis, a patient with a higher propensity to get LAMA treatment also has a higher risk for poor outcomes, as seen when stratified by propensity quantile. Rates for mortality (12.2\%) and COPD-related rehospitalization (14.6\%) for days 31-365 post-discharge in our study population demonstrate how fragile these patients are.

As we performed an observational study, we may not have been able to adjust fully for confounding, despite using IPTW to minimize selection biases. There may still be unadjusted residual confounding relating to indication bias in our data, muting beneficial outcomes for LAMA [61]. Findings have been shown to vary slightly when different causal inference methods are utilized in analyses [47]. Given the differences we observed across quintiles in our stratified model, a matched analysis of the data that by definition would not use the entire sample would undoubtedly produce different findings. However, findings from a matched analysis would only be generalized to a population similar to the matched population [47]. Spirometry information was not available to assess obstruction severity in patients, and even though we included other indicators of more advanced respiratory disease, such as exacerbations, oxygen use, and reliever medication use, these are proxy measures for disease severity.

Our study reviewed outcomes associated with receipt of a maintenance therapy within the first 30 days post-discharge. Thirty days was chosen to allow patients sufficient time to fill a prescription received at discharge or during a follow-up physician visit. Bias from immortal time can be present in a retrospective observational cohort study when the individuals in one exposure group that meet the inclusion criteria cannot, by definition, have had an event [62, 63]. For example, individuals in our study must have been alive to receive a LAMA prescription at discharge or during the following 30 days. We do not believe that this 30-day period used to determine exposure affected our study findings. In our study, the same 30-day period was used to determine exposure for both groups, and the follow-up period to assess outcomes did not begin at discharge but at day 31 . Individuals with outcomes occurring during the 30-day exposure period were excluded. Prior to imposing this exclusion criterion, there was no difference in the rate of all-cause 30-day readmissions between the LAMA and no LABD treatment groups. The no LABD treatment group did have a higher mortality rate in the first 30 days post-discharge (1.4\% versus $0.6 \%)$, but the effect size difference was low $(\mathrm{ES}=0.08)$.

We utilized an intent-to-treat (ITT) approach in our analysis. Results from an ITT may be confounded when a substantive portion of a no-treatment group later initiates treatment. The overwhelming majority of patients in the no LABD group in our study did not claim for LAMA or any LABD in the follow-up period. In the period from 31 days to 180 days post-discharge, only $3 \%$ of the no LABD group had a prescription for any LABD. Baker and colleagues observed similar results for the follow-up post-discharge period among those who did not receive LABD therapy during the initial hospitalization for COPD [54]. Among the LAMA users in this study, the mean LAMA PDC value at 180 days indicated $50 \%$ usage and the mean PDC value declined to $<50 \%$ in the last half of the year. However, during this period, persistence with LAMA therapy was higher among individuals who did not have a COPD-related readmission or die.

Drug therapy on hand was determined using prescription claims data. There is a possibility that we were missing prescription information on some Medicare enrollees, but estimates in this study are comparable to other analyses. Adherence and persistence with COPD treatments have repeatedly been found to be suboptimal [41, 64, 65]. Among Medicare enrollees, adherence to any COPD maintenance treatment has remained fairly unchanged, averaging approximately 55\% from 2008 through 2013 [66]. RCTs have demonstrated that LAMA treatment can significantly reduce the risk for exacerbations, but suboptimal persistence may be a factor behind the less than strong association between LAMA use and COPD-related readmission determined in this observational retrospective study.

Follow-up with a physician within 30 days of discharge has been associated with fewer exacerbation-related readmissions [67], and was not reviewed in this analysis. Early assessment and subsequent follow-up allows a multipronged approach to preventing readmission that includes recommended interventions such as bronchodilators (LAMAs, LABAs, LAMA/LABAs) as well as nonpharmaceutical 
interventions (smoking cessation, rehabilitation) to be devised [67]. COPD-related hospitalizations are common and costly. Such traits imply that there is great potential benefit in encouraging providers to prescribe these treatments after discharge and in encouraging patients to maintain adherence to the therapy in the long term.

\section{Conclusion}

Our analysis demonstrates that LABD treatment is significantly effective at reducing COPD-related rehospitalizations. The reduction in risk in this population was small $(95 \% \mathrm{CI}$ $0.86,0.98)$ and weaker than that observed in RCTs among individuals with $\geq 1$ prior exacerbation $(95 \%$ CI $0.72,1.00$ ) $[24,25]$, which is not unexpected in a real-world analysis. Persistence with therapy was suboptimal among patients prescribed LAMA therapy, and efforts to improve adherence to therapy could potentially improve the real-world effectiveness of LAMA for patients.

Acknowledgements The authors meet the criteria for authorship as recommended by the International Committee of Medical Journal Editors (ICMJE).

Author Contributions MHR, DWM, and HP provided substantial contributions to the conception and design of the work, the acquisition, analysis, or interpretation of data for the work, and to drafting the work and revising it critically for important intellectual content; they also provided final approval of the version to be published and agree to be accountable for all aspects of the work in ensuring that questions related to the accuracy or integrity of any part of the work are appropriately investigated and resolved.

\section{Compliance with Ethical Standards}

Funding This investigator-initiated study was financially supported by Boehringer Ingelheim Pharmaceuticals, Inc. (BIPI). BIPI had no role in the design, analysis, or interpretation of the results in this study; however, BIPI was given the opportunity to review the manuscript for medical and scientific accuracy as it relates to BIPI substances, as well as intellectual property considerations.

Conflict of interest M. Roberts received grant funding from Boehringer Ingelheim Pharmaceuticals for this study. She has additionally received grant funding (outside of this study) from GlaxoSmithKline, AstraZeneca, Pfizer, and Endo Pharmaceuticals. D. Mapel received grant funding from Boehringer Ingelheim Pharmaceuticals for this study and has served as consultant to Boehringer Ingelheim Pharmaceuticals, GlaxoSmithKline, Mylan, and Theravance Biopharma. H. Petersen is retired from Lovelace Respiratory Research Institute and reports no conflict of interest.

Open Access This article is distributed under the terms of the Creative Commons Attribution-NonCommercial 4.0 International License (http://creativecommons.org/licenses/by-nc/4.0/), which permits any noncommercial use, distribution, and reproduction in any medium, provided you give appropriate credit to the original author(s) and the source, provide a link to the Creative Commons license, and indicate if changes were made.

\section{References}

1. HCUP Fast Stats. Healthcare Cost and Utilization Project (HCUP). Most common diagnoses for inpatient stays. Rockville: Agency for Healthcare Research and Quality; 2019. https://www. hcup-us.ahrq.gov/faststats/national/inpatientcommondiagnoses. jsp. Accessed 16 May 2019.

2. McGhan R, Radcliff T, Fish R, Sutherland ER, Welsh C, Make B. Predictors of rehospitalization and death after a severe exacerbation of COPD. Chest. 2007;132(6):1748-55. https://doi. org/10.1378/chest.06-3018.

3. Baker CL, Zou KH, Su J. Risk assessment of readmissions following an initial COPD-related hospitalization. Int J Chron Obstruct Pulmon Dis. 2013;8:551-9. https://doi.org/10.2147/COPD.S5150 7

4. Genao L, Durheim MT, Mi X, Todd JL, Whitson HE, Curtis LH. Early and long-term outcomes of older adults after acute care encounters for chronic obstructive pulmonary disease exacerbation. Ann Am Thorac Soc. 2015;12(12):1805-12. https://doi. org/10.1513/AnnalsATS.201504-250OC.

5. Soriano JB, Kiri VA, Pride NB, Vestbo J. Inhaled corticosteroids with/without long-acting $\beta$-agonists reduce the risk of rehospitalization and death in COPD patients. Am J Respir Med. 2003;2(1):67-74.

6. Harries TH, Thornton H, Crichton S, Schofield P, Gilkes A, White PT. Hospital readmissions for COPD: a retrospective longitudinal study. NPJ Prim Care Respir Med. 2017;27(1):31. https://doi. org/10.1038/s41533-017-0028-8.

7. Schiotz M, Price M, Frolich A, Sogaard J, Kristensen JK, Krasnik A, et al. Something is amiss in Denmark: a comparison of preventable hospitalisations and readmissions for chronic medical conditions in the Danish healthcare system and Kaiser Permanente. BMC Health Serv Res. 2011;11:347. https://doi. org/10.1186/1472-6963-11-347.

8. Groenewegen KH, Schols AM, Wouters EF. Mortality and mortality-related factors after hospitalization for acute exacerbation of COPD. Chest. 2003;124(2):459-67.

9. Almagro P, Calbo E, Ochoa de Echaguen A, Barreiro B, Quintana $\mathrm{S}$, Heredia JL, et al. Mortality after hospitalization for COPD. Chest. 2002;121(5):1441-8.

10. Slenter RH, Sprooten RT, Kotz D, Wesseling G, Wouters EF, Rohde GG. Predictors of 1-year mortality at hospital admission for acute exacerbations of chronic obstructive pulmonary disease. Respiration. 2013;85(1):15-26. https://doi.org/10.1159/00034 2036.

11. Ho TW, Tsai YJ, Ruan SY, Huang CT, Lai F, Yu CJ, et al. In-hospital and one-year mortality and their predictors in patients hospitalized for first-ever chronic obstructive pulmonary disease exacerbations: a nationwide population-based study. PLoS One. 2014;9(12):e114866. https://doi.org/10.1371/journal.pone.0114866.

12. García-Sanz María-Teresa MT, Canive-Gomez J-C, Senin-Rial L, Aboal-Vinas J, Barreiro-Garcia A. One-year and long-term mortality in patients hospitalized for chronic obstructive pulmonary disease. J Thorac Dis. 2017;9(3):636-45.

13. Lindenauer PK, Dharmarajan K, Qin L, Lin Z, Gershon AS, Krumholz HM. Risk trajectories of readmission and death in the first year after hospitalization for chronic obstructive pulmonary disease. Am J Respir Crit Care Med. 2017;197(8):1009-17. https ://doi.org/10.1164/rccm.201709-1852OC. 
14. CMS. Readmissions Reduction Program. http://www.cms.gov/ Medicare/Medicare-Fee-for-Service-Payment/AcuteInpatientP PS/Readmissions-Reduction-Program.html. Accessed 28 June 2015.

15. Fingar KR, Washington R. Trends in hospital readmissions for four high-volume conditions, 2009-2013: statistical brief \#196. Healthcare Cost and Utilization Project (HCUP) statistical briefs. Rockville: Agency for Healthcare Research and Quality; 2015. https://www.hcup-us.ahrq.gov/reports/statbriefs/sb196-Readm issions-Trends-High-Volume-Conditions.jsp. Accessed 12 Mar 2019.

16. Roberts MH, Clerisme-Beaty E, Kozma CM, Paris A, Slaton T, Mapel DW. A retrospective analysis to identify predictors of COPD-related rehospitalization. BMC Pulm Med. 2016;16(1):68. https://doi.org/10.1186/s12890-016-0231-3.

17. Global Initiative for Chronic Obstructive Lung Disease (GOLD). Global strategy for the diagnosis, management, and prevention of COPD (2019 report). https://goldcopd.org/wp-content/uploa ds/2018/11/GOLD-2019-v1.7-FINAL-14Nov2018-WMS.pdf. Accessed 12 Mar 2019.

18. Alagha K, Palot A, Sofalvi T, Pahus L, Gouitaa M, Tummino $\mathrm{C}$, et al. Long-acting muscarinic receptor antagonists for the treatment of chronic airway diseases. Ther Adv Chronic Dis. 2014;5(2):85-98. https://doi.org/10.1177/2040622313518227.

19. Global Initiative for Chronic Obstructive Lung Disease (GOLD). Global strategy for the diagnosis, management, and prevention of COPD (updated 2011). http://www.goldcopd.org. Accessed 8 May 2012.

20. NCQA. Healthcare Effectiveness Data and Information Set (HEDIS) measures and technical resources. Effectiveness of care. Pharmacotherapy management of COPD exacerbation. https:// www.ncqa.org/hedis/measures/pharmacotherapy-management -of-copd-exacerbation/. Accessed 12 Mar 2019.

21. US Food and Drug Administration. Approval letter for SPIRIVA ${ }^{\circledR}$ HandiHaler $^{\circledR}$ (tiotropium bromide inhalation powder). NDA 21-395. Washington, DC: U.S. Food and Drug Administration; 2004. http://www.accessdata.fda.gov/drugsatfda_docs/apple tter/2004/21395ltr.pdf. Accessed 26 Oct 2018.

22. US Food and Drug Administration. Efficacy supplement letter for SPIRIVA ${ }^{\circledR}$ HandiHaler $^{\circledR}$ (tiotropium bromide inhalation powder). NDA 21-395/S-029. Washington, DC: U.S. Food and Drug Administration; 2009. http://www.accessdata.fda.gov/drugsatfda _docs/appletter/2009/021395s0291tr.pdf. Accessed 26 Oct 2018.

23. Halpin D, Menjoge S, Viel K. Patient-level pooled analysis of the effect of tiotropium on COPD exacerbations and related hospitalisations. Prim Care Respir J. 2009;18(2):106-13. https://doi. org/10.4104/pcrj.2009.00017.

24. Karner C, Chong J, Poole P. Tiotropium versus placebo for chronic obstructive pulmonary disease. Cochrane Database Syst Rev. 2012;7:CD009285. https://doi.org/10.1002/14651858.cd009285. pub2.

25. Karner C, Chong J, Poole P. Tiotropium versus placebo for chronic obstructive pulmonary disease. Cochrane Database Syst Rev. 2014. https://doi.org/10.1002/14651858.cd009285.pub3.

26. D'Urzo A, Ferguson GT, van Noord JA, Hirata K, Martin C, Horton R, et al. Efficacy and safety of once-daily NVA237 in patients with moderate-to-severe COPD: the GLOW1 trial. Respir Res. 2011;12:156. https://doi.org/10.1186/1465-9921-12-156.

27. Ni H, Soe Z, Moe S. Aclidinium bromide for stable chronic obstructive pulmonary disease. Cochrane Database Syst Rev. 2014;9:CD010509. https://doi.org/10.1002/14651858.cd010509. pub2.

28. Halpina DMG. Lessons from the major studies in COPD: problems and pitfalls in translating research evidence into practice. Prim Care Respir J. 2010;19:170. https://doi.org/10.4104/ pcrj.2010.00015.
29. Mapel DW, Roberts MH. Management of asthma and chronic obstructive pulmonary disease with combination inhaled corticosteroids and long-acting beta-agonists: a review of comparative effectiveness research. Drugs. 2014;74(7):737-55. https://doi. org/10.1007/s40265-014-0214-8.

30. Lederer DJ, Bell SC, Branson RD, Chalmers JD, Marshall R, Maslove DM, et al. Control of confounding and reporting of results in causal inference studies. Guidance for authors from editors of respiratory, sleep, and critical care journals. Ann Am Thorac Soc. 2019;16(1):22-8. https://doi.org/10.1513/annal sats.201808-564ps.

31. Feemster LC, Au DH. Penalizing hospitals for chronic obstructive pulmonary disease readmissions. Am J Respir Crit Care Med. 2014;189(6):634-9. https://doi.org/10.1164/rccm.201308-1541PP.

32. Press VG, Au DH, Bourbeau J, Dransfield MT, Gershon AS, Krishnan JA, et al. Reducing chronic obstructive pulmonary disease hospital readmissions. An official American Thoracic Society workshop report. Ann Am Thorac Soc. 2019;16(2):161-70. https ://doi.org/10.1513/annalsats.201811-755ws.

33. Rubin DB. Estimating causal effects of treatments in randomized and nonrandomized studies. J Educ Psychol. 1974;66(5):688-701. https://doi.org/10.1037/h0037350.

34. Roberts M, Mapel D, Borrego M, Raisch D, Georgopoulos L, van der Goes D. Severe COPD exacerbation risk and long-acting bronchodilator treatments: comparison of three observational data analysis methods. Drugs Real World Outcomes. 2015;2(2):16375. https://doi.org/10.1007/s40801-015-0025-6.

35. Cox DR, Oakes D. The scope of survival analysis: 1.3. Censoring. In: Analysis of survival data. Boca Raton: Chapman \& Hall; 1984. p. 4-5.

36. Austin PC, Lee DS, Fine JP. Introduction to the analysis of survival data in the presence of competing risks. Circulation. 2016;133(6):601-9. https://doi.org/10.1161/CIRCULATIO NAHA.115.017719.

37. Robins JM, Hernan MA, Brumback B. Marginal structural models and causal inference in epidemiology. Epidemiology. 2000;11(5):550-60. https://doi.org/10.1097/00001648-20000 9000-00011.

38. Fine JP, Gray RJ. A proportional hazards model for the subdistribution of a competing risk. J Am Stat Assoc. 1999;94(446):496509. https://doi.org/10.1080/01621459.1999.10474144.

39. Elixhauser A, Steiner C, Harris DR, Coffey RN. Comorbidity measures for use with administrative data. Med Care. 1998;36(1):8-27.

40. Klabunde CN, Potosky AL, Legler JM, Warren JL. Development of a comorbidity index using physician claims data. J Clin Epidemiol. 2000;53(12):1258-67.

41. Mapel DW, Dutro MP, Marton JP, Woodruff K, Make B. Identifying and characterizing COPD patients in US managed care. A retrospective, cross-sectional analysis of administrative claims data. BMC Health Serv Res. 2011;11:43. https://doi. org/10.1186/1472-6963-11-43.

42. Grosso LM, Lindenauer P, Wang C, Savage S, Potteiger J, Geary LL, et al. Hospital-level 30-day readmission following admission for an acute exacerbation of chronic obstructive pulmonary disease. Measure methodology report. New Haven: Yale New Haven Health Services Corporation/Center for Outcomes Research \& Evaluation (YNHHSC/CORE); 2011. https ://www.cms.gov/Medicare/Quality-Initiatives-Patient-Asses sment-Instruments/HospitalQualityInits/Measure-Methodolog y.html Accessed 12 Mar 2019.

43. Cohen J. Things I have learned (so far). Am Psychol. 1990;45(12):1304-12. https://doi.org/10.1037/0003-066X.45.12.1304.

44. Cohen J. A power primer. Psychol Bull. 1992;112(1):155-9. 
45. D’Agostino RB. Propensity score methods for bias reduction in the comparison of a treatment to a non-randomized control group. Stat Med. 1998;17(19):2265-81.

46. Rosenbaum PR, Rubin DB. Constructing a control-group using multivariate matched sampling methods that incorporate the propensity score. Am Stat. 1985;39(1):33-8.

47. Austin PC, Mamdani MM. A comparison of propensity score methods: a case-study estimating the effectiveness of postAMI statin use. Stat Med. 2006;25(12):2084-106. https://doi. org/10.1002/sim.2328.

48. Normand SLT, Landrum NB, Guadagnoli E, Ayanian JZ, Ryan TJ, Cleary PD, et al. Validating recommendations for coronary angiography following acute myocardial infarction in the elderly: a matched analysis using propensity scores. J Clin Epidemiol. 2001;54(4):387-98.

49. Hosmer D, Lemeshow S. Applied logistic regression. 2nd ed. New York: Wiley; 2000.

50. Rosenbaum PR, Rubin DB. The central role of the propensity score in observational studies for causal effects. Biometrika. 1983;70(1):41-55.

51. Cox DR, Oakes D. Analysis of survival data. Boca Raton: Chapman \& Hall; 1984.

52. StataCorp. Stata 15 survival analysis reference manual: stcrreg-competing-risks regression. College Station: Stata Press; 2014. https://www.stata.com/manuals/ststcrreg.pdf. Accessed 3 Nov 2016.

53. Cochran WG. Effectiveness of adjustment by subclassification in removing bias in observational studies. Biometrics. 1968;24(2):295. https://doi.org/10.2307/2528036.

54. Baker Christine LC, Zou KH, Su J. Long-acting bronchodilator use after hospitalization for COPD: an observational study of health insurance claims data. Int J Chron Obstruct Pulmon Dis. 2014;9:431-9.

55. NHLBI. Morbidity \& mortality: 2012 chart book on cardiovascular, lung, and blood diseases. Bethesda: National Institutes of Health National Heart, Lung and Blood Institute; 2012. http:// www.nhlbi.nih.gov/research/reports/2012-mortality-chart-book. Accessed 28 June 2015.

56. Stuart BC, Simoni-Wastila L, Zuckerman IH, Davidoff A, Shaffer T, Yang HW, et al. Impact of maintenance therapy on hospitalization and expenditures for Medicare beneficiaries with chronic obstructive pulmonary disease. Am J Geriatr Pharmacother. 2010;8(5):441-53. https://doi.org/10.1016/j.amjop harm.2010.10.002.

57. Lindenauer PK, Shieh MS, Pekow PS, Stefan MS. Use and outcomes associated with long-acting bronchodilators among patients hospitalized for chronic obstructive pulmonary disease. Ann Am
Thorac Soc. 2014;11(8):1186-94. https://doi.org/10.1513/Annal sATS.201407-3110C.

58. Kirchmayer U, Cascini S, Agabiti N, Di Martino M, Bauleo L, Formoso G, et al. One-year mortality associated with COPD treatment: a comparison of tiotropium and long-acting beta2agonists in three Italian regions: results from the OUTPUL study. Pharmacoepidemiol Drug Saf. 2016;25(5):578-89. https://doi. org/10.1002/pds.3961.

59. Trudo F, Kern DM, Davis JR, Tunceli O, Zhou S, Graham EL, et al. Comparative effectiveness of budesonide/formoterol combination and tiotropium bromide among COPD patients new to these controller treatments. Int J Chron Obstruct Pulmon Dis. 2015;10:2055-66. https://doi.org/10.2147/COPD.S90658.

60. Oba Y, Keeney E, Ghatehorde N, Dias S. Dual combination therapy versus long-acting bronchodilators alone for chronic obstructive pulmonary disease (COPD): a systematic review and network meta-analysis. Cochrane Database Syst Rev. 2018. https ://doi.org/10.1002/14651858.cd012620.pub2.

61. Salas M, Hofman A, Stricker BHC. Confounding by indication: An example of variation in the use of epidemiologic terminology. Am J Epidemiol. 1999;149(11):981-3.

62. Suissa S. Immortal time bias in observational studies of drug effects. Pharmacoepidemiol Drug Saf. 2007;16(3):241-9.

63. Lévesque LE, Hanley JA, Kezouh A, Suissa S. Problem of immortal time bias in cohort studies: example using statins for preventing progression of diabetes. BMJ. 2010;340:b5087.

64. Make B, Dutro MP, Paulose-Ram R, Marton JP, Mapel DW. Undertreatment of COPD: a retrospective analysis of US managed care and Medicare patients. Int J Chron Obstruct Pulmon Dis. 2012;7:1-9.

65. Restrepo RD, Alvarez MT, Wittnebel LD, Sorenson H, Wettstein $\mathrm{R}$, Vines DL, et al. Medication adherence issues in patients treated for COPD. Int J Chron Obstruct Pulmon Dis. 2008;3(3):371-84.

66. Nishi SP, Maslonka M, Zhang W, Kuo Y-F, Sharma G. Pattern and adherence to maintenance medication use in Medicare beneficiaries with chronic obstructive pulmonary disease: 2008 2013. Chronic Obstr Pulm Dis. 2018;5(1):16-26. https://doi. org/10.15326/jcopdf.5.1.2017.0153.

67. Global Initiative for Chronic Obstructive Lung Disease (GOLD). Chapter 5: management of exacerbations: Hospital discharge and follow-up. In: Global strategy for the diagnosis, management, and prevention of COPD (2019 Report). Fontana: GOLD; 2019. https ://goldcopd.org/wp-content/uploads/2018/11/GOLD-2019-v1.7FINAL-14Nov2018-WMS.pdf. Accessed 12 Mar 2019. 